Goce Spasovski

Raymond Vanholder

Bruno Allolio

Djillali Annane

Steve Ball

Daniel Bichet

Guy Decaux

Wiebke Fenske

Ewout J. Hoorn

Carole Ichai

Michael Joannidis

Alain Soupart

Robert Zietse

Maria Haller

Sabine van der Veer

Wim Van Biesen

Evi Nagler

\title{
Erratum to: Clinical practice guideline
on diagnosis and treatment of hyponatraemia \\ Erratum to: Clinical practice guideline
on diagnosis and treatment of hyponatraemia
}

Published online: 25 March 2014

(C) Springer-Verlag Berlin Heidelberg and ESICM 2014

The online version of the original article can be found under doi: 10.1007/s00134-014-3210-2.

\section{G. Spasovski}

State University Hospital Skopje, Skopje, Macedonia

\section{R. Vanholder · W. Van Biesen ( )}

Ghent University Hospital, Ghent, Belgium

e-mail: Wim.VanBiesen@UGent.be

B. Allolio - W. Fenske

Würzburg University Hospital, Würzburg, Germany

\section{Annane}

Raymond Poincaré Hospital, University of Versailles

Saint Quentin, Paris, France

\section{S. Ball}

Newcastle Hospitals and Newcastle University, Newcastle, UK

D. Bichet

Consultant Nephrologist, Sacré-Coeur Hospital,

University of Montreal, Montreal, Canada

G. Decaux · A. Soupart

Erasmus University Hospital, Brussels, Belgium
E. J. Hoorn · R. Zietse

Erasmus Medical Centre, Rotterdam, The Netherlands

C. Ichai

Nice University Hospital, Nice, France

M. Joannidis

Innsbruck University Hospital, Innsbruck, Austria

M. Haller · S. van der Veer $\cdot$ E. Nagler

ERBP Methods Support Team, Ghent University Hospital,

Ghent, Belgium

M. Haller

KH Elisabethinen Linz, Linz, Austria

S. van der Veer

Centre for Informatics, Amsterdam Medical Centre,

Amsterdam, The Netherlands

\section{Erratum to: Intensive Care Med (2014) 40:320-331 DOI 10.1007/s00134-014-3210-2}

The name of the co-author Ewout J. Hoorn was rendered incorrectly in the manuscript supplied for publication. 